\title{
Análise de Custo-benefício de Rede Óptica WDM com Agregação de Tráfego e Agregação de Comprimentos de Onda
}

\author{
Helvécio M. Almeida, Eduardo M. G. de Queiroz e Amílcar C. César
}

\begin{abstract}
Resumo - Uma análise de custo-benefício de rede óptica WDM é realizada neste artigo. A arquitetura dos nós permite realizar agregação de comprimentos de onda (waveband grooming-WBG) na camada óptica e agregação de tráfego (traffic grooming-TG) em camada eletrônica. O algoritmo proposto combina roteamento com TG e WBG com "granularidade" uniforme ou não-uniforme. A relação custo-benefício é baseada no custo de várias configurações de nós e no número de solicitações de conexão atendidas. As simulações são realizadas com base na topologia da rede NSFNet e os resultados obtidos mostram a utilidade da análise para a escolha adequada de configuração de nós.
\end{abstract}

Palavras-chave - WDM, RWA, agregação de tráfego, agregação de comprimento de onda, relação custo-benefício.

Abstract - This paper relates a cost-benefit analysis of WDM optical network. The network nodes perform uniform or nonuniform waveband grooming (WBG) and traffic grooming (TG). The cost-benefit analysis is based on the cost of some node architectures using WBG and TG and on the successful connection requests. The simulations of the algorithm were carried out on the NSFNet topology. Numerical results showed how useful the analysis can be to choose the best node configuration.

Index Terms - WDM, RWA, uniform and non-uniform waveband grooming, traffic grooming, cost-benefit analysis.

\section{INTRODUÇÃO}

A infra-estrutura formada pelas redes ópticas utilizando técnica WDM (wavelength division multiplexing) tem sido constantemente aperfeiçoada para satisfazer a demanda de largura de faixa, oriunda principalmente dos serviços multimídia e do grande número de usuários [1]. À camada óptica têm sido incorporados dispositivos de alto desempenho e funções antes restritas às camadas superiores da rede. Por outro lado, as várias redes, em geral suportando protocolos e taxas de transmissão diversos, têm sido interligadas, formando malha com ampla cobertura terrritorial. Sendo assim, esforços têm sido despendidos para manter as redes técnica e economicamente viáveis.

As redes ópticas são formadas por nós interligados por enlace de fibra óptica. Os nós têm a função de rotear tráfego por meio de crossconnects ópticos (OXCs). A comutação totalmente óptica tem sido explorada como alternativa para a

Helvécio M. Almeida, Eduardo M. G. de Queiroz, Amílcar C. César, Universidade de São Paulo, Escola de Engenharia de São Carlos, Depto. Eng. Elétrica, São Carlos, SP. E-mails: asah@terra.com.br; \{equeiroz;amilcar\}@ sel.eesc.usp.br.

Pesquisa parcialmente financiada pelo CNPq, CAPES, FAPESP (Projeto Tidia-KyaTera, Lab. LightWays, proc. 03/08269-7) e CPqD, FINEP, FUNTTEL (Projeto Giga, contrato 1360). comutação elétrica que contribui para limitar a taxa de transmissão. Os OXCs podem dispor de grande número de portas para reduzir a probabilidade de bloqueio de solicitações de conexão. Entretanto, esta alternativa eleva os custos da rede.

Considerando recursos da rede óptica como comprimentos de onda $(\lambda s)$, largura de faixa e portas de OXCs, o uso de técnicas eficientes de gerenciamento destes recursos pode melhorar a relação custo-benefício [2]. O objetivo é atender as solicitações de conexão originadas nos nós com baixa probabilidade de bloqueio utilizando o menor número possível de recursos da rede. Para alcançar este objetivo podemos combinar algoritmo de roteamento e alocação de $\lambda$ (routing and wavelength assignment-RWA) com as técnicas de agregação de tráfego (traffic grooming-TG) e agregação de comprimentos de onda (waveband grooming-WBG).

O RWA consiste em rotear tráfego pelos vários enlaces conectando os nós origem e destino por meio de único ou diferentes $\lambda s$ [3].

WBG é uma técnica atraente para reduzir o número de portas dos OXCs. Ela consiste em agregar vários comprimentos de onda formando um feixe e roteá-los em grupo pela rede como se fossem um único $\lambda$ [4]. $O$ emprego desta técnica melhora a relação custo-benefício, embora aumente levemente a probabilidade de bloqueio global [5], [6]. Os nós da rede equipados com WBG podem agrupar $\lambda \mathrm{s}$ de duas formas: uniforme, se os nós WBG agrupam quantidade iguais de $\lambda$, e não-uniforme, se as quantidades de $\lambda$ são distintas. A agregação não-uniforme é mais flexível em relação a quantidade de $\lambda$ s agregados [2].

Camadas de rede como a IP (Internet protocol)/MPLS (multiprotocol label switching) geram tráfego de baixas taxas de transmissão, enquanto a camada óptica suporta tráfego de altas taxas, como OC-48 (2,5 Gbps), OC-192 (10 Gbps), ou OC-768 (40 Gbps). Os tráfegos de baixas taxas precisam ser eficientemente agregados nos comprimentos de onda. Esta é a técnica TG [7], que tem sido realizada nas camadas eletrônicas da rede, via conversão óptica-eletrônica-óptica (OEO).

Sendo assim, o TG é uma técnica eficiente de uso da largura de faixa disponível em um $\lambda$, mas exibe a desvantagem de utilizar conversão OEO. Por outro lado, a técnica WBG reduz o número de portas utilizadas nos OXCs, mas pode aumentar levemente a probabilidade de bloqueio. Portanto, um compromisso entre o uso dos comprimentos de onda, largura de faixa, portas de OXCs e conversões OEO é crucial para atender a demanda dos usuários e manter a rede técnica e economicamente viável. A medida deste compromisso é a relação custo-benefício, entendida como a 
relação entre o custo de uso de equipamentos (ou recursos) e o número de solicitações de conexão atendidas.

As técnicas TG [7]-[13] e WBG não-uniforme [4], [12] já foram tratadas isoladamente na literatura, bem como análise de custo-benefício de uso de portas de OXCs incluindo WBG uniforme e não-uniforme [2]. Em [12], WBG não-uniforme foi tratado em conjunto com agregação IP para a investigação dos efeitos dos esquemas de agregação sobre a topologia e o custo da rede. Entretanto, nesse trabalho não foi tratada a relação entre a agregação uniforme e não-uniforme com a probabilidade de bloqueio e a relação custo-benefício.

A contribuição deste artigo reside na realização de análise de custo-benefício de rede óptica WDM utilizando simultaneamente WBG uniforme ou não-uniforme e TG. Na análise de custo-benefício realizada, quatro configurações foram analisadas, sendo duas com TG e WBG não-uniforme, uma com TG e WBG uniforme e uma com TG sem WBG. Para todas as configurações analisadas são mostradas as probabilidades de bloqueio e a relação custo-benefício.

Um controle de admissão de chamada (CAC) com base em demanda de largura de faixa e no algoritmo first-fit [10] é proposto neste artigo para analisar as configurações. Os resultados de simulação de desempenho do algoritmo na topologia da rede NSFNet [11] mostram a utilidade da análise para escolher arquitetura de nó com relação custobenefício adequada.

Este artigo está organizado da seguinte forma. Na Seção II é feita a formulação do problema, descritas as arquiteturas dos nós da rede e a proposta do CAC. Na Seção III é proposta uma análise de custos da arquitetura de nós. Na Seção IV são apresentados e comentados os resultados da simulação e na Seção V são apresentadas as conclusões.

\section{FORMULAÇÃO DO PROBLEMA}

Dada uma solicitação de conexão $i$ entre os nós $a$ e $b$, o CAC procura o caminho mais curto e que disponha de largura de faixa disponível para atender a solicitação e não considera conversão de comprimento de onda.

\section{A. Arquitetura dos Nós}

Os nós da rede são compostos por OXCs, que exibem custos menores que os equivalentes eletrônicos [14]. Os nós também são dotados de transmissores e receptores para realizar conversão OEO e permitir a realização de agregação de tráfego.

Um OXC é capaz de comutar, adicionar e retirar $\lambda \mathrm{s}$. Em [14] os autores utilizaram o OXC multigranular (MG)-OXC que, além de executar as funções convencionais dos OXCs, também pode comutar, adicionar e retirar grupos de comprimentos de onda (wavebands-WBs). O conceito de "granularidade" significa agregação de um certo número de $\lambda \mathrm{s}$ em uma única porta. Assim, a "granularidade" é a quantidade de $\lambda s_{(k)}$ que são agregados em um $W B, W_{k}$.

Um caminho $W B$ ocupa apenas duas portas (entrada e saída) de um MG-OXC em um nó. Os caminhos $W B$ podem ser comutados opticamente como uma unidade. Dessa maneira, quando se agregam $\lambda \mathrm{s}$ em um MG-OXC o número de portas necessárias para se comutar $\lambda s$ individuais é reduzido.
Os nós utilizados neste artigo são formados pela combinação de MG-OXCs com capacidade de agregação nãouniforme e uniforme de $\lambda \mathrm{s}$ e OXCs convencionais com capacidade de TG (via conversão OEO), sendo: nós com capacidade de TG sem WB (OXC) e nós MG-OXCi, nos quais $i=2$ a 6 indica a "granularidade". A Fig. 1 mostra a arquitetura dos nós para dois wavebands.
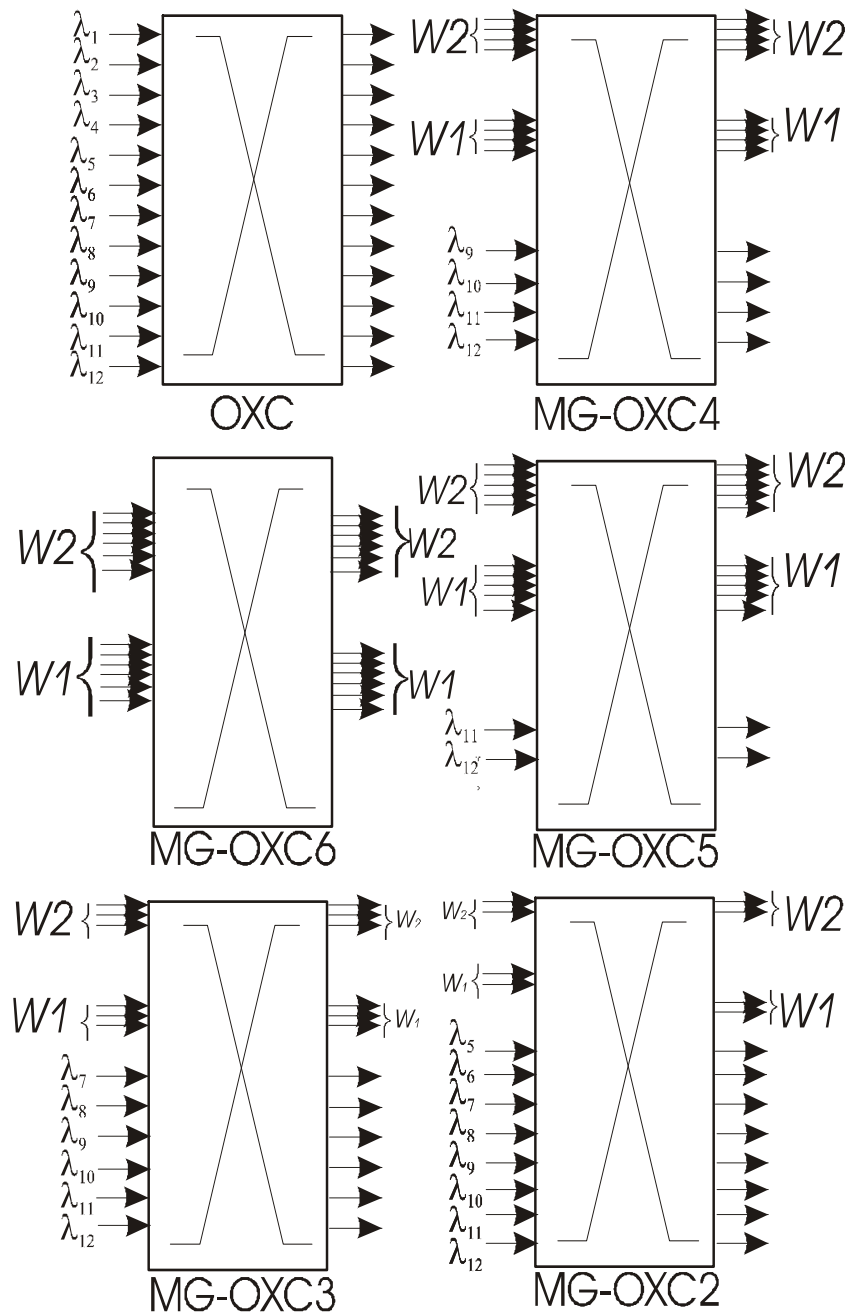

Figura 1. Nós $M G$-OXCs com diferentes valores de "granularidade".

Os nós MG-OXC recebem via algoritmo uma solicitação de conexão de um $\lambda$ não-agregado ao $W B$ e a largura de faixa disponível é verificada. Caso não haja largura de faixa disponível, a solicitação é bloqueada. Na ocorrência de uma alocação do tipo $W B$, o algoritmo verifica a largura de faixa disponível no $W B$ e, caso não haja largura de faixa disponível, bloqueia a solicitação.

Os nós OXC recebem via algoritmo uma solicitação de $\lambda \mathrm{e}$ a largura de faixa disponível neste $\lambda$ é verificada para aceitar ou não a solicitação de conexão.

A Tabela I mostra as larguras de faixa adotadas e as probabilidades de geração (chegada) de cada tipo de solicitação de conexão.

Em todos os tipos de nós, o TG é realizado em cada $\lambda$ nãoagregado ao WB. O TG é realizado com base no fator de TG, $G_{a}$, que é a quantidade de chamadas que podem ser agrupadas em um determinado $\lambda$. Por exemplo, um $\lambda$ de largura de faixa máxima igual a 2,5 Gbps e um fator de TG igual a 16 pode agregar no máximo 16 conexões de largura de faixa de 155,52 
Mbps. Portanto, o fator de TG pode variar de 1 a 16 para as situações relacionadas na Tabela I.

TABELA I

LARGURAS DE FAIXA DAS SOLICITAÇÕES DE CONEXÃO

\begin{tabular}{|c|c|c|}
\hline $\begin{array}{c}\text { Tipo de } \\
\text { Solicitação }\end{array}$ & $\begin{array}{c}\text { Largura de Faixa } \\
(\mathrm{Mbps})\end{array}$ & $\begin{array}{c}\text { Probabilidade de } \\
\text { Geração (\%) }\end{array}$ \\
\hline I & 155,52 & 59 \\
\hline II & 622,08 & 15 \\
\hline III & 933,12 & 10 \\
\hline IV & 1.244 & 8 \\
\hline V & 1.866 & 5 \\
\hline VI & 2.488 & 3 \\
\hline
\end{tabular}

Desta maneira, se a largura de faixa e o fator de TG de um $\lambda$ suportar a solicitação, então ela é aceita. Dados $\lambda_{a}$ e $G_{a}$, a solicitação será aceita apenas se a condição de disponibilidade de largura de faixa for satisfeita e o número de conexões presentes em $\lambda_{a}\left(G_{e}\right)$ for $G_{e} \leq G_{a}$. A Fig. 2 mostra o esquema de conexões dos nós com e sem WB.

Conexão WaveBand

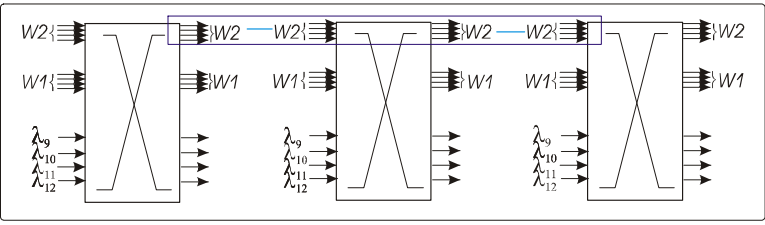

Conexão Wavelength

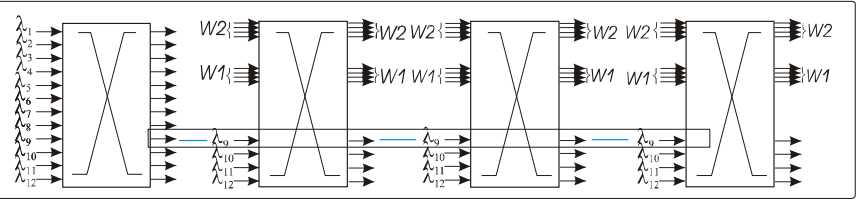

Figura 2. Exemplo de conexões com nós com ou sem waveband.

\section{B. Controle de Admissão de Chamadas}

O controle de admissão de chamadas proposto utiliza WBG e o principal parâmetro desta técnica é a "granularidade". Uma "granularidade" 2 indica que dois $\lambda$ s serão agregados e tratados como único. A capacidade de largura de faixa da agregação é equivalente à capacidade dos dois $\lambda$ s na qual as solicitações de conexão serão alocadas. Assim, serão alocadas tantas solicitações quanto possíveis nessa largura de faixa equivalente.

Os nós da rede óptica simulada geram dois tipos de solicitação de conexão: as conexões WB e as não-WB, que não utilizam WB e são alocadas em um $\lambda$. Para que ocorra uma conexão do tipo WB é necessário que todos os nós participantes da conexão sejam do tipo WB. Para as conexões que não utilizam WB, o caminho óptico pode conter nós do tipo MG-OXC, pois, neste caso, utilizam os comprimentos de onda não-agregados.

Dada uma solicitação $i$ entre os nós $a$ e $b$, as seguintes situações podem ocorrer na tentativa de conexão entre os nós $j$ e $k$ pertencentes ao caminho $(a, b)$ :

1. Nó $j$ não é $W B$ e inicia a conexão. O algoritmo escolhe um $\lambda_{\mathrm{e}}$ de acordo com a política first-fit [10], ou seja, utiliza o primeiro $\lambda$ que dispõe de capacidade de largura de faixa para aceitar a solicitação. O nó $j$ verifica se há disponibilidade de alocar a solicitação $i$ em $\lambda_{\text {e }}$ para o estabelecimento da solicitação com o nó $k$.
2. Nó $j$ é $W B$, mas utiliza $\lambda$ s não-agregados. Aloca uma solicitação em determinado comprimento de onda $\lambda_{\mathrm{e}}$ e verifica se a capacidade de largura de faixa é suficiente para aceitar a solicitação $i$. Se for, então aceita a solicitação entre os nós $j$ e $k$.

3. Nó $j$ é $W B$ e todos os nós do caminho $(a, b)$ também são. Ele verifica se há largura de faixa disponível para alocar a solicitação $i$ no $W B W_{e}$.

Nos três casos, qualquer negação de alocação entre os nós $j$ e $k$ devido à inexistência de largura de faixa suficiente resulta em bloqueio de solicitação. O fluxograma do CAC é exibido na Fig.4.

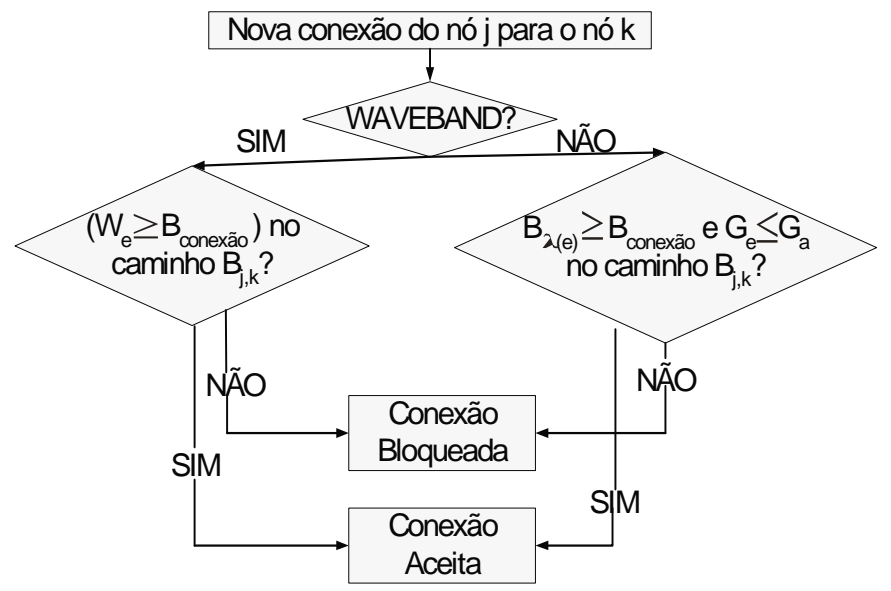

Figura 4. Fluxograma do controle de admissão de chamadas (CAC).

\section{ANÁliSE dO CUSTO DOS NÓS}

Neste artigo, o MG-OXC é usado como referência de custo. Desta forma, uma arquitetura totalmente óptica com funcionalidade $W B G$ presente no nó exibiria o menor custo. A adição gradativa de nós com OXCs e capacidade TG encarecem a arquitetura.

Dada a dificuldade em estabelecer custos efetivos para os equipamentos utilizados nos nós da rede, também adotamos neste artigo a abordagem feita em [14], que é a de avaliar custos relativos entre os equipamentos. Assim, o custo total da arquitetura de nós é calculado de acordo com os fatores:

1. Nós MG-OXC6 (fator 6): 3,0 por porta;

2. Nós MG-OXC5 (fator 5): 2,5 por porta;

3. Nós MG-OXC4 (fator 4): 2,0 por porta;

4. Nós MG-OXC3 (fator 3): 1,5 por porta

5. Nós MG-OXC2 (fator 2): 1,0 por porta

6. Nós OXCs com capacidade de TG: 4,0 por porta.

A combinação de dispositivos para formar as arquiteturas dos nós pode ser feita considerando o número de $\lambda$ s que foram agregados em WBs. Por exemplo, em um nó com $12 \lambda$ s e 2 WBs de "granularidade" 4, $8 \lambda$ s estão agrupados em 2 grupos de 4 . Os $4 \lambda$ s restantes não estão agrupados e podem ou não agregar tráfego via TG. Se forem 2 WBs com "granularidade" $5,10 \lambda$ s estão agrupados em 2 grupos de 5 e os $2 \lambda$ s restantes podem ou não agregar tráfego via TG. Portanto, para os 12 comprimentos de onda considerados, a Fig.5 ilustra os custos das portas e a Tabela II mostra os valores dos custos dos nós considerados no sistema.

A relação custo-benefício é calculada de acordo com: 


$$
C / B=\frac{\text { custo total do conjunto de nós }}{\text { conexões aceitas }}
$$

na qual o custo total do conjunto de nós equivale à soma do custo de cada nó utilizado.

É de se esperar que (1) tenha o menor valor possível, significando o maior número possível de solicitações de conexão atendidas com mínimo de recursos.

TABELA II

TIPO DE NÓ, "GRANULARIDADE” E CUSTO

\begin{tabular}{|c|c|c|}
\hline Tipo do Nó & "Granularidade" & Custo \\
\hline MG-OXC & 4 & 20,0 \\
\hline MG-OXC & 6 & 6,0 \\
\hline MG-OXC & 5 & 13,0 \\
\hline MG-OXC & 3 & 27,0 \\
\hline MG-OXC & 2 & 34,0 \\
\hline OXC & - & 48,0 \\
\hline
\end{tabular}
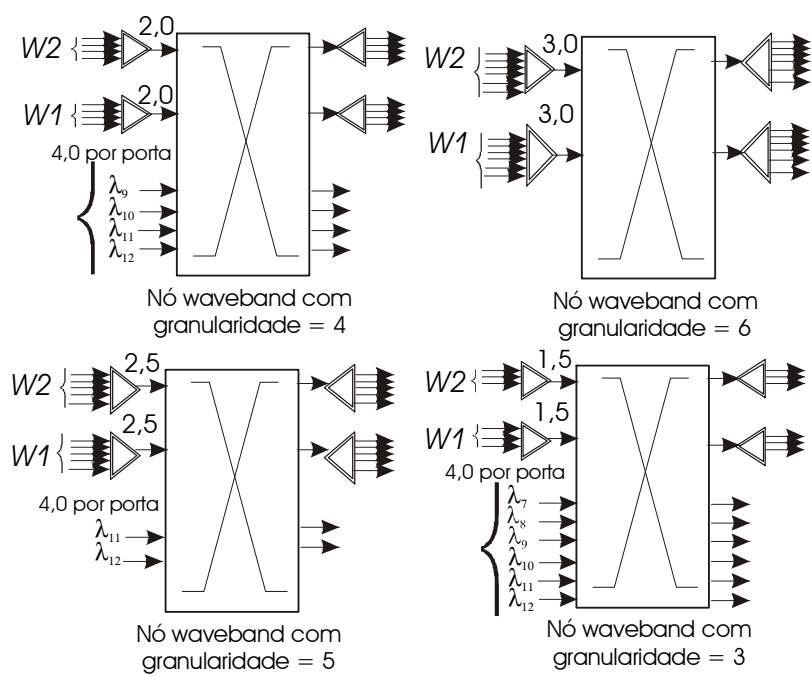

granularidade $=6$
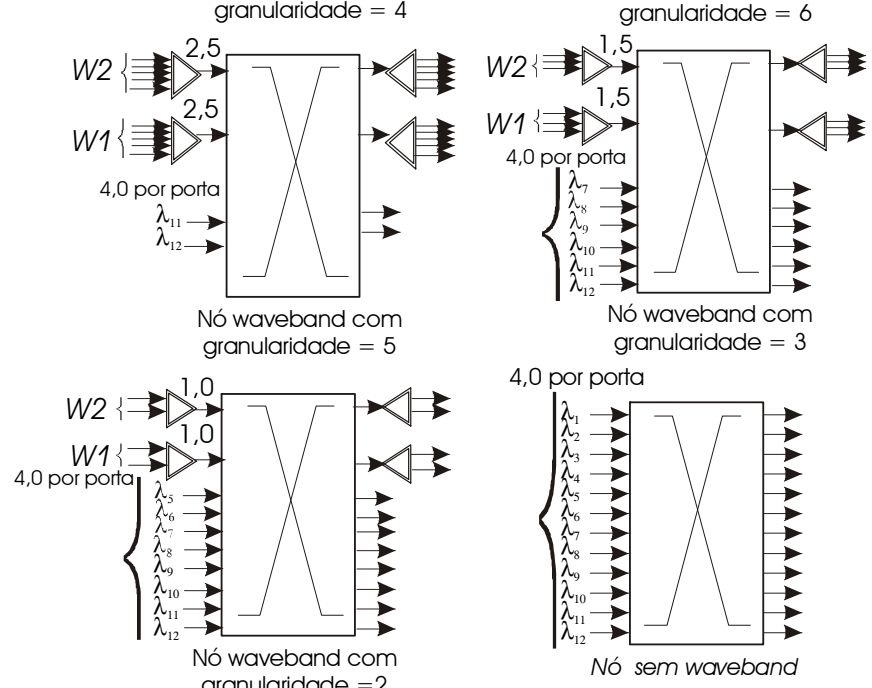

4,0 por porta

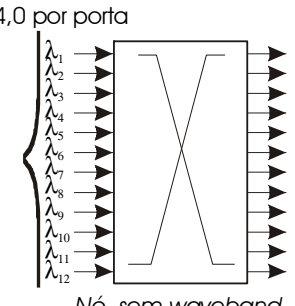

Nó sem woveband

Figura 5. Custo associado a cada porta das arquiteturas dos nós.

\section{RESUltAdOS NUMÉRICOS}

Nesta seção são apresentados os resultados obtidos por meio de simulações com base na topologia da rede NSFNeT [11], mostrada na Fig.6. Os nós em destaque (cor azul) são os que possuem funcionalidade WB. Eles foram escolhidos para formar uma sequiência entre os dois extremos da rede, dado que uma conexão WB somente é estabelecida quando todos os nós participantes são MG-OXC.

Quatro configurações foram simuladas e em todas elas consideramos 2 WBs:

1. Nós WB (6, 7, 8, 9, 10 e 13) com capacidade de agregação igual a 4 em todos os nós (configuração uniforme), ou seja, MG-OXC4 e com capacidade de TG nos $\lambda$ s nãoagregados. O restante dos nós é OXCs. Custo total da rede: $6($ nós MG-OXC $) \times 20,0+8($ nós $\mathrm{OXC}) \times 48,0=504,0$;
2. Nós OXCs sem WB e com capacidade TG. Custo total da rede: 672,0

3. Nós WB $(6,7,8,9,10$ e 13) com as seguintes capacidades de agregação: MG-OXC4 nos nós 6,7,9 e 13, MG-OXC5 no nó 8, MG-OXC3 no nó 10 e TG nos $\lambda$ s nãoagregados. O restante dos nós é OXCs. Custo total da rede: 506,0 ;

4. Nós WB $(6,7,8,9,10$ e 13) com as seguintes capacidades de agregação: MG-OXC4 nos nós 6, 7, 9 e 13, MG-OXC6 no nó 8 e MG-OXC2 no nó 10 e TG de $\lambda$ s nãoagregados e o restante dos nós é OXCs. Custo total da rede: 506,0.

Para cada configuração foram simuladas 100.000 solicitações de conexão. As simulações foram realizadas em computador Pentium 4 com 2,8 GHz de clock e 2,0 Gbytes de memória RAM. Para gerar cada ponto das curvas foram gastos, em média, 2 horas e 30 minutos e o modelo do tráfego utilizado é de Poisson.

A Fig.7 mostra as probabilidades de bloqueio das diferentes configurações. A configuração 4 apresenta a maior probabilidade de bloqueio, pois além da "granularidade" igual a 4 nos nós 6, 7, 9 e 13 e "granularidade" igual a 2 no nó 10 , ela reúne no nó 8 todos o $\lambda$ s agregados em WB, ou seja, não há $\lambda$ disponível para realizar outro tipo de conexão a não ser WB, o que limita sua capacidade de conexão com outros nós que disponham de $\lambda \mathrm{s}$.

No outro extremo, as configurações 2 e 3 apresentam as menores probabilidades de bloqueio. $\mathrm{Na}$ configuração 2 observa-se que não há $\lambda s$ agregados formando WBs e, portanto, todos os $\lambda$ s estão disponíveis para realizar conexão com outros nós. Assim, nota-se que a probabilidade de bloqueio é a menor dentre todas as configurações. A configuração 3 possui "granularidade" igual a 4 nos nós 6,7 , 9 e 13, "granularidade" igual a 3 no nó 10 e "granularidade" igual a 5 no nó 8 e sua probabilidade de bloqueio tem valor semelhante ao da configuração 2, pois o nó 8 não possui mais a restrição de não dispor de $\lambda$ s para realizar a conexão.

A configuração 1 mostrou-se um pouco pior que as 2 e 3 com relação aos valores das probabilidades de bloqueio porque no nó 10 existem mais $\lambda$ s agregados ao $\mathrm{WB}$ do que na configuração 3. Desta maneira, o nó 10 mostra-se mais sensível do que os outros nós à diminuição do número de $\lambda$ s que estão disponíveis para conexões não-WBs. Este fato está relacionado ao maior número de enlaces que existem no nó 10 em relação aos outros nós WBs.

A Fig.8 mostra a relação custo-benefício das configurações simuladas. A configuração 2 apresenta a maior relação custo-benefício por não possuir nós com capacidade de realizar WB, mas também possui a menor probabilidade de bloqueio. As configurações 1 e 3 apresentam a melhor relação custo-benefício e também valores de probabilidade de bloqueio semelhantes, sendo que a configuração 3 apresenta o menor valor. Por fim, a configuração 4 apresenta o maior valor de probabilidade de bloqueio e valores de custo um pouco mais alto que as configurações 3 e 4 .

Desta maneira, a técnica WB é útil porque diminui o custo dos nós e, ao mesmo tempo, não muda de maneira significativa a probabilidade de bloqueio de conexões quando os nós que possuem WB são bem ajustados com relação à "granularidade". 


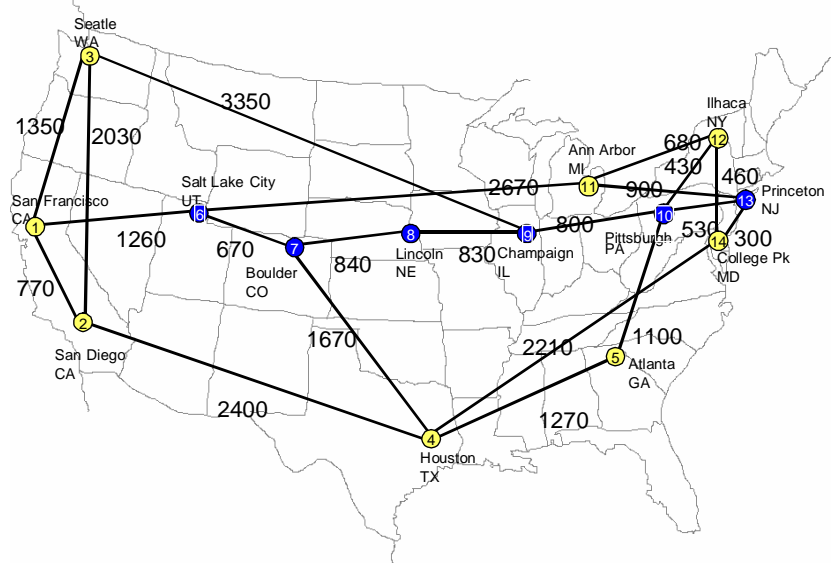

Figura.6. Topologia da rede NSFNeT [11]. Os nós em azul realizam waveband.

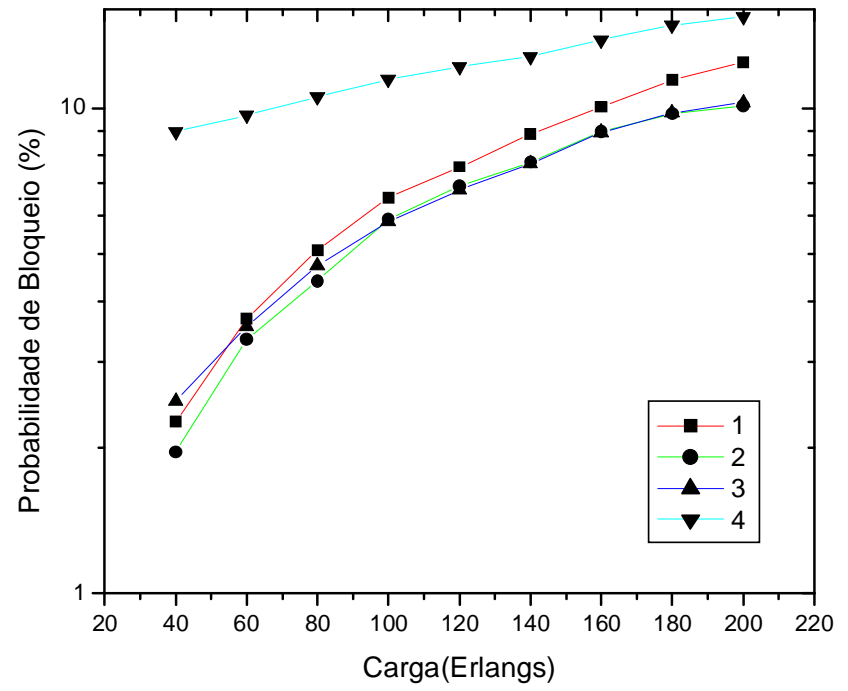

Figura 7. Probabilidade de bloqueio de tráfego das configurações simuladas. Ver configurações dos nós no início da Seção IV.

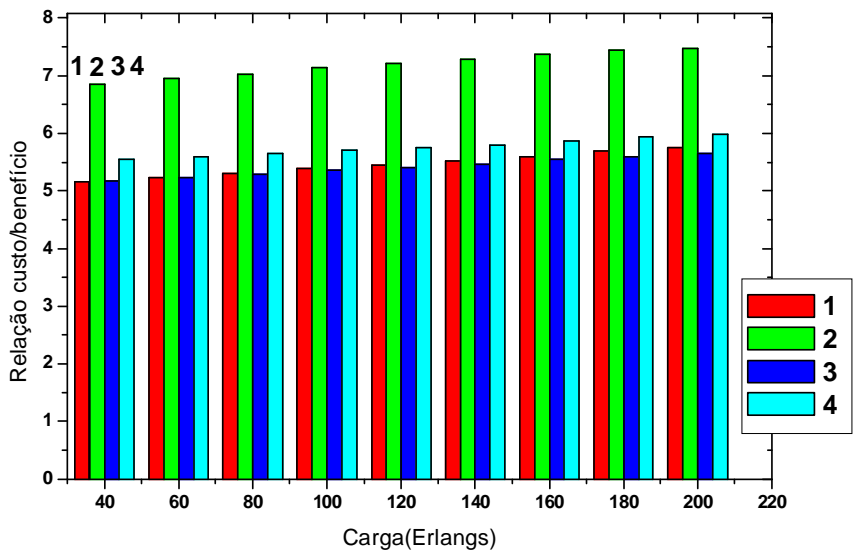

Figura 8. Relação custo-benefício das configurações simuladas. Ver configurações dos nós no início da Seção IV.

\section{CONCLUSÕES}

Neste artigo foi utilizada a técnica agregação waveband uniforme e não-uniforme em conjunto com a agregação de tráfego para analisar a relação custo-benefício com base na topologia da rede NSFNeT [11].

A vantagem da técnica WBG é realizar agregação de $\lambda$, reduzindo o número de portas dos OXCs e, conseqüentemente, o custo.

Os resultados mostram uma melhora significativa na relação custo-benefício das configurações 1 e 3 , indicando que, apesar do aumento da probabilidade de bloqueio de conexões produzido pelo $\mathrm{WBG}$, há benefício porque o número de solicitações atendidas aumenta. É possível concluir também que com uma variação não-uniforme de "granularidade" nos nós que realizam WB pode-se amenizar o aumento da probabilidade de bloqueio e, ainda assim, manter custo mais baixo com relação à configuração que utiliza apenas OXCs.

\section{REFERÊNCIAS}

[1]R. Ramaswami, and K.N. Sivarajan, K. N., Optical Networks: A Practical Perspective, Morgan Kaufmann Publishers, 1998.

[2]R. Izmailov, S. Ganguly,V. Kleptsyn, A.C.Varsou "Non-Uniform Waveband Hierarchy in Hybrid Optical Networks ", Proceedings of IEEE INFOCOM 2003.

[3]X. Zhang and C. Qiao, "Wavelength Assignment in Fixed Routing WDM Networks,” Proc., ICC'97, Montreal , Canada, vol.1, pp. 406-410, junho 1997.

[4]R. Lingampalli and P. Vengalam, "Effect of wavelength and waveband grooming on an all-optical networks with single layer photonic switching," Proceedings of OFC 2002.

[5]Y. Suemura, I. Nishioka, Y. Maeno and S. Araki, "Routing of hierarchical paths in an optical network", Proceedings of APCC 2001.

[6]Y. Suemura, I. Nishioka, Y. Maeno, S. Araki, R. Izmailov, S. Ganguly "Hierarchical routing in layered ring and mesh optical networks", Proceedings of IEEE ICC 2001.

[7]R. Dutta e G.N. Rouskas, "Traffic Grooming in WDM Networks: Past and Future", IEEE Network, pp. 46-56, novembro/dezembro de 2002.

[8]K. R. Beny and E. Modiano, "Reducing electronic multiplexing costs in SONET/WDM rings with dynamic changing traffic," IEEE Journal on Selected Areas in Communications, Vol 18, No. 10, pp. 19611971,2000

[9]A. Chiu and E. Modiano, "Traffic grooming algorithms for reducing electronic multiplexing costs in WDM ring networks," Journal of Lightwave Technology, Vol. 18, No. 1, pp.2-12,2000.

[10] R. E. Wagner, R. C. Alferness, A. A. M. Saleh, and M. S. Goodman, "MONET: Multiwavelength optical networking," J. Lightwave Technol., vol. 4, pp. 1349-1355, junho 1996.

[11] B Mukherjee, Optical WDM Networks, Springer Science+Bussines Media, Inc, 2006.

[12] R. Parthiban, R.S. Tucker and C.Leckie. "Waveband Grooming and IP Aggregation in Optical Networks", J. of Ligtwave Technology, vol 21,no. 11, pp.2476-2488, novembro 2003.

[13] M.A.C. Lima, A.C. César e A.F.R. Araújo, "Optical Network Optimization with Transmission Impairments Based on Genetic Algorithm", 2003 SBMO/IEEE MTT-S International Microwave and Optoelectronics Conference- IMOC 2003 Proc., vol. 1, pp. 361-365, Foz do Iguaçu, Brasil, 20 a 23 de setembro de 2003.

[14] M.Li, B. Ramamurthy "Heterogeneous waveband switching in wavelength division multiplexed networks based on autonomous clustering architecture (invited)," J. of Optical Networking.,vol 5, no.9, pp.667-680, setembro 2006. 